\title{
PADRÃO DE MOTIVAÇÃO E DESFECHOS DE PROGRESSO ACADÊMICO: ESTUDO LONGITUDINAL COM ESTUDANTES DE MEDICINA
}

\section{Dejano Tavares Sobral}

CDD: 370.154

\begin{abstract}
RESUMO
O propósito do estudo foi analisar a motivação educacional de estudantes de medicina em relação com indicadores de progresso acadêmico durante a graduação na Universidade de Brasília. A Escala de Motivação Acadêmica foi aplicada em turmas consecutivas ao total de 285 participantes no início do segundo ano. Foram analisados estatisticamente indicadores da Escala e suas correlações prospectivas com índices de rendimento acadêmico e realização de monitoria, e também diferenças nesses índices entre quatro grupos de configuração motivacional identificados em análise classificatória. Os resultados mostraram correlações positivas e significantes entre motivação intrínseca e rendimento acadêmico, bem como entre realização de monitoria e combinação de motivação intrínseca e regulação externa. Grupos com orientação motivacional expressamente autônoma ou mista (autônoma e controlada) realizaram número significantemente maior de monitorias do que grupos com menor autodeterminação motivacional. Os achados são discutidos - a luz da teoria da autodeterminação - quanto aos aspectos de adaptação motivacional e de influências de transição da ambiência educacional. Concluindo, os resultados sugerem que indicadores de motivação caracterizados no início da graduação têm efeito preditivo pequeno, mas significante no desempenho acadêmico continuado e no exercício eletivo de monitoria. Esse desfecho eletivo diferiu significantemente entre estudantes com distintos perfis motivacionais.
\end{abstract}

\section{PALAVRAS-CHAVE}

Motivação; Monitoria; Avaliação da aprendizagem; Rendimento escolar; Escala de motivação acadêmica

\section{MOTIVATION PATTERNS AND OUTCOMES OF ACADEMIC PROGRESS: A LONGITUDINAL STUDY WITH MEDICAL STUDENTS}

\begin{abstract}
The purpose of this work was to analyze medical students 'motivation features in relation to outcomes of academic progress during undergraduate studies. The Academic Motivation Scale (AMS) was administered in consecutive classes to a total of $2852^{\text {nd }}$ year students who graduated at the University of Brasilia. Statistical analyses were undertaken on AMS indicators and their correlations with cumulative GPA and peer-tutoring outcomes, as well as on outcome differences among four motivation-profile groups identified in cluster analysis. The results showed positive, significant correlations between intrinsic motivation components and GPA, as well as between peer-tutoring activity and a mix of intrinsic motivation and extrinsic motivation by external regulation. Cluster analysis groups with definite autonomous motivation or with combined (autonomous and controlled) motivation undertook more extended peer tutoring than those with less self-determined patterns. The discussion appraises the findings in the light of the SelfDetermination Theory as related to aspects of motivation adaptation and influences of transition in educational context. In conclusion, the results suggest that motivational features ascertained at the beginning of the medical program have a significant, albeit small, predictive effect on the students' achievement and peer tutoring activity. The later outcome differed significantly among students with distinguishing motivational configurations.
\end{abstract}

\section{KEYWORDS}

Educational measurement; Motivation; Peer tutoring; Achievement; Academic motivation scale 


\section{PESQUISA \\ Área Temática: Ensino de Psicologia \& Psicologia Educacional Número Especial: Motivação}

\section{INTRODUÇÃO}

Motivação, no campo educacional, é um construto complexo que abrange conotações diversas em relação ao direcionamento e à energia na aprendizagem. A motivação dos aprendizes se diferencia - segundo o modelo hierárquico - em relação ao grau e tipo, bem como ao nível de generalidade. O grau implica a intensidade motivacional, o tipo se refere ao porquê da motivação, isto é, as atitudes e os propósitos subjacentes, enquanto o nível de generalidade diz respeito à esfera de expressão, isto é, global, contextual e situacional (VALLERAND; RATELLE, 2002).

O modelo hierárquico se baseia na teoria da autodeterminação, a qual identifica três categorias básicas de motivação: (a) motivação intrínseca, em que se faz algo pelo interesse e prazer inerentes à ação, ressalvando-se que as pessoas diferem quanto ao seu interesse por distintas atividades; (b) motivação extrínseca, em que se faz algo por razões instrumentais, diferentes do interesse intrínseco na ação; e (c) desmotivação (amotivation), em que não se percebe contingência entre atos e efeitos, implicando ações sem intencionalidade (DECI; RYAN, 1991; 2000; 2008). Segundo proposição daquela teoria, os diversos tipos de orientação motivacional diferem quanto ao grau de autodeterminação, em que motivação intrínseca representa o grau mais elevado e desmotivação o mais baixo. A motivação extrínseca, sobretudo, tem facetas que subentendem níveis variáveis de autonomia em termos de grau de internalização e integração de valores e regulação de condutas. Outra proposição postula que fatores do contexto psicossocial que dão suporte a necessidades psicológicas básicas - autonomia, competência e vínculo - promovem ação intencional ou motivada do tipo intrínseco, bem como facilitam a internalização e integração de fatores associados à motivação extrínseca (DECI; RYAN, 1991; 2000; 2008; RYAN; DECI, 2000). Além disso, há evidências que percepções de autonomia, competência e vínculo medeiam as influências de fatores da ambiência psicossocial na autodeterminação da motivação em três esferas de generalidade: global, contextual e situacional (VALLERAND, 1997; 2002; VALLERAND; RATELLE, 2002).

Guay et al. (2008) afirmaram - entre outras conclusões baseadas na revisão da extensa literatura internacional sobre estudos educacionais ligados à teoria da autodeterminação - que quanto maior o endosso das formas de motivação autônoma, melhor 
os estudantes aprendem, mais positivas e persistentes são suas vivências e maiores suas realizações acadêmicas. Entretanto, esses autores ressalvaram que estudantes podem endossar, com sucesso, motivos intrínsecos (autônomos) e extrínsecos (controlados) em seus estudos, na dependência de características pessoais e de influências da ambiência acadêmica. Boruchovitch (2008) fez ilações do mesmo teor em breve síntese sobre os achados principais da revisão da literatura nacional sobre motivação na educação.

O estudo pioneiro de Willians e Deci (1996) explicitou a relevância dos princípios da teoria de autodeterminação para a educação médica. Os autores mostraram que situações específicas da formação podem favorecer diferencialmente a expressão de motivação autônoma (primariamente intrínseca) ou de motivação controlada (extrínseca) nos estudantes de medicina, com efeitos nas atitudes na aprendizagem e na relação com pacientes. Maior suporte da autonomia dos aprendizes pelos docentes favoreceria entendimento mais profundo, equilíbrio psicológico e orientação humanística (WILLIANS ; DECI, 1998). Misch (2002), entretanto, questionou a hipótese andragógica de que estudantes de medicina teriam predominância de motivação autônoma e sugeriu que inexiste resposta simples para a questão de motivação para aprender em virtude da interação mútua e frequente de fatores internos e externos na formação médica. Em trabalho anterior, Mann (1999) já postulava que motivações intrínsecas e extrínsecas eram complementares na formação médica, aduzindo que os estudantes de medicina reagiriam ao contexto complexo de incentivos e barreiras que podem ter influência discrepante nas atitudes e condutas dos aprendizes. Em tese, portanto, o endosso de valores manifestos nos diversos cenários da formação profissional acarretaria efeitos na expressão dos tipos primários de motivação e no aprendizado realizado.

Nessa perspectiva, o espectro de motivação de estudantes de medicina em Brasília foi examinado em linha de estudos de observação com utilização da versão em Português de l'Échelle de Motivation en Éducation - Escala de Motivação Acadêmica (SOBRAL, 2003; 2004; 2008). Esse instrumento visa avaliar a motivação habitual dos estudantes na esfera contextual da educação e se baseia nos princípios da teoria da autodeterminação (VALLERAND et al., 1989; VALLERAND, 1997). Análises das respostas ao instrumento por indivíduos de diferentes faixas etárias e origem geográfica - têm revelado propriedades fidedignas de consistência interna, estabilidade temporal e validade de construto (VALLERAND et al., 1992; 1993; FAIRCHILD et al., 2005). Nos estudos com a Escala de 
Motivação Acadêmica, referidos acima, os dados de revalidação condizem em larga margem com tais características psicométricas. Ademais, as respostas dos estudantes mostraram predominância de motivação autônoma e revelaram associações significantes e peculiares dos componentes do instrumento aos fatores pessoais - isto é, autoconfiança como aprendiz, orientação de estudo significativa e reflexão na aprendizagem - e aos fatores situacionais, expressos nos índices de valoração da vivência no curso (SOBRAL, 2003; 2004). Os achados do terceiro estudo indicaram inter-relações significantes entre fatores pessoais e situacionais na atribuição do grau de autodeterminação da motivação e, paralelamente, na intenção de adesão ao curso. Em particular, observou-se um perfil diferencial na relação do índice de autodeterminação da motivação acadêmica com motivos precedentes da escolha de medicina os quais têm diferido seletivamente entre grupos de estudantes (SOBRAL, 2008).

Duas questões serviram de incentivo para a continuação da linha de estudos, ainda utilizando a Escala de Motivação Acadêmica. A primeira diz respeito ao impacto em longo prazo dos indicadores de motivação nos índices objetivos de realização ou rendimento acadêmico - aspecto em que há discrepância na literatura (FAIRCHILD et al., 2005; GUAY et al., 2008). A segunda questão contempla as diferenças nesses índices de progresso entre estudantes agrupados conforme o perfil motivacional - no enfoque centrado na pessoa, segundo a denominação de Ratelle et al. (2007). Achados anteriores evidenciaram, por meio de análise classificatória, configurações distintas de motivação autônoma e motivação controlada entre grupos de estudantes de medicina. Esses grupos diferiram em diversos atributos de aprendizagem (SOBRAL, 2004).

No estudo corrente, dois índices de desfecho serviram de indicadores de progresso: o rendimento escolar - como sinalizador crítico da aprendizagem - e o exercício de monitoria ao longo do curso. Levou-se em conta, na escolha de monitoria, que o acesso a essa atividade é opcional e bem diversificado na instituição, propiciando senso de escolha e iniciativa aos aprendizes. Ao término do primeiro ano do curso, estudantes (da mesma população do estudo atual) têm identificado motivos diversos para realização de monitoria em disciplinas, dentre os quais predominam o fortalecimento de entendimento e habilidades, a sondagem de área de opção de especialização e as oportunidades para trabalhar com determinados docentes. 
O presente trabalho tem os seguintes objetivos:

1. Descrever as características motivacionais dos estudantes de medicina segundo os indicadores da Escala de Motivação Acadêmica (EMA).

2. Analisar as relações dos indicadores da EMA, como fatores antecedentes, com desfechos acadêmicos representados por exercício de monitoria em disciplinas e rendimento cumulativo da aprendizagem no curso.

3. Averiguar os perfis motivacionais aflorando na amostra do estudo e como os estudantes agrupados nesses perfis diferiram em termos dos desfechos acadêmicos.

\section{MÉTODO}

\section{Participantes}

A população alvo do estudo foi o alunado do curso de medicina da Universidade de Brasília. O critério de inclusão no estudo foi o acesso ao terceiro período do fluxo curricular, por amostragem consecutiva envolvendo todos os estudantes matriculados em turmas sucessivas ao longo de quatro anos. No conjunto total de 296 estudantes $(56,5 \%$ homens) havia maioria (62\%) de oriundos do próprio Distrito Federal. Ao ingressar no curso, a média de idade dos estudantes era $19,3(\mathrm{dp}=1,57)$, variando de 16 a 30 anos. Foram excluídos das análises finais 11 estudantes (cinco mulheres) por causa de evasão antes da conclusão do curso, causada por descumprimento de requisitos acadêmicos, mudança de curso, ou transferência para outra instituição.

\section{Procedimentos}

A coleta de dados em cada turma foi realizada no início do terceiro período do fluxo curricular. Os dados foram obtidos com asseguramento expresso de confidencialidade individual e as respostas coletivas foram comentadas com os participantes no contexto de orientação educativa. 


\section{Instrumento e Medidas}

Escala de Motivação Acadêmica (EMA). O instrumento utilizado no estudo incluiu os 28 itens da versão original - que representam razões para o estudante vir à universidade - além de escala analógica de intenção (motivação global para fazer os esforços) de prosseguir os estudos e concluir o curso (SOBRAL, 2008). Os itens estão organizados em sete componentes: três correspondem a formas de motivação intrínseca; outros três incorporam formas de motivação extrínseca e, o último, representa desmotivação. A caracterização desses componentes e suas correlações com a escala de intenção de concluir o curso são relacionadas a seguir.

(a) Motivação intrínseca para saber - significa fazer algo pelo prazer que decorre de aprender, explorar ou entender; $r=0,429(\mathrm{P}<0,001)$.

(b) Motivação intrínseca para realização - compreende participar de atividade pela satisfação que decorre da realização ou criação de alguma coisa; $r=0,317(\mathrm{P}<0,001)$.

(c) Motivação intrínseca para vivenciar estímulos - envolve fazer algo por causa das sensações estimulantes derivadas da participação; $r=0,407(\mathrm{P}<0,001)$.

(d) Motivação por identificação - implica fazer algo porque valoriza e considera importante fazê-lo; $r=0,429(\mathrm{P}<0,001)$.

(e) Motivação por introjeção - subentende fazer algo porque se pressiona a si próprio a fazê-lo; $\mathrm{r}=-0,034(\mathrm{P}=0,570)$.

(f) Regulação externa - subentende fazer algo porque se sente pressionado por outros a fazê-lo, seja por recompensas ou restrições; $r=-0,055(\mathrm{P}=0,353)$.

(g) Desmotivação - expressa ausência de percepção de contingências entre as ações e seus desfechos ou falta de intenções e desestímulo; $r=-0,583(P<0,001)$.

Outros quatro indicadores combinados foram calculados e são identificados abaixo, junto às respectivas correlações com a escala de intenção de concluir o curso.

(h) Motivação autônoma - corresponde à soma dos componentes de motivação intrínseca com motivação extrínseca por identificação; $r=0,446(\mathrm{P}=<0,001)$.

(i) Motivação controlada - corresponde à soma de regulação por introjeção e regulação externa; $\mathrm{r}=-0,051(\mathrm{P}=0,390)$. 
(j) Motivação total - corresponde à soma dos indicadores de motivação autônoma e de motivação controlada; $r=0,264(\mathrm{P}=<0,001)$.

(k) Índice de autodeterminação da motivação (IAM) - representa o espectro de autodeterminação, integrando os escores dos sete componentes da EMA em medida única; $\mathrm{r}=$ 0,620 $(\mathrm{P}<0,001)$. Calculou-se esse índice mediante a fórmula IAM $=[(2(a+b+c) / 3+d)-$ $((\mathrm{e}+\mathrm{f}) / 2+2 \mathrm{~g})]$ em que as letras correspondem aos componentes da EMA (GUAY et al., 2003).

Desfechos. Dois indicadores de progresso na aprendizagem dos estudantes foram utilizados: o índice de rendimento acadêmico e o índice de exercício de monitoria. O índice de rendimento - que é atualizado cumulativamente ao término de cada período letivo corresponde à média ponderada das notas finais, na escala de zero a cinco, de todas as disciplinas já cursadas pelo estudante. A faixa numérica desse índice varia de zero a 5,00.

O índice de exercício de monitoria corresponde ao número total de monitorias realizadas entre o $2^{\circ}$ e o $12^{\circ}$ período do fluxo curricular do curso (uma por semestre), em disciplinas obrigatórias ou optativas já cursadas com aprovação. A faixa numérica da atividade varia, portanto, de zero a 11.

\section{Análise}

Os textos de Howell (2002) e de Tabachnick e Fidell (2007) orientaram os planos e procedimentos de análise. Os procedimentos estatísticos principais incluíram análises de correlação, regressão e variância, utilizando General Linear Procedure, e, também, análise classificatória, mediante o algoritmo de conjunto k-means, precedido de análise de aglomeração hierárquica e conferindo os cuidados recomendados (CLATWORTHY et al., 2005). Todos os procedimentos foram efetuados no pacote SPSS, versão 10. Os escores dos indicadores da EMA (componentes simples ou combinados, exceto o índice de autodeterminação da motivação) foram expressos em escalas de sete pontos, para clareza e consistência. 


\section{RESULTADOS}

A Tabela 1 apresenta os dados de consistência interna (alfa de Cronbach), médias (escala de sete pontos) e intercorrelações dos componentes da EMA, nas respostas dos participantes. Todos os componentes mostraram consistência aceitável (alfa >0,70). As médias mais elevadas foram para motivação para saber $(6,00 \mathrm{dp}=0,87)$ e motivação por identificação $(6,22 \mathrm{dp}=0,83)$, enquanto o menor foi para desmotivação $(1,41 \mathrm{dp}=0,65)$. As correlações entre componentes de motivação intrínseca e extrínseca foram positivas e significantes, mas de magnitude variável conformando o continuum previsto na teoria de autodeterminação, exceto principalmente na correlação elevada entre motivação para realização e introjeção. As correlações de desmotivação foram significantes e negativas com os componentes de motivação intrínseca e por identificação, mas não foram significantes com aqueles de motivação por introjeção e regulação externa.

\section{TABELA 1}

Coeficientes de correlação (r), médias e desvios padrões, e coeficientes de consistência interna (alfa de Cronbach, na diagonal) para componentes da Escala de Motivação Acadêmica - EMA ( $N=285)$

\begin{tabular}{|c|c|c|c|c|c|c|c|}
\hline Componentes da EMA & 1 & 2 & 3 & 4 & 5 & 6 & 7 \\
\hline 1. Intrínseca - saber & $(0,86)$ & 0,585 & 0,575 & 0,420 & 0,192 & 0,173 & $-0,403$ \\
\hline 2. Intrínseca - realização & & $(0,82)$ & 0,586 & 0,293 & 0,571 & 0,259 & $-0,200$ \\
\hline 3. Intrínseca - estímulo & & & $(0,74)$ & 0,377 & 0,314 & 0,160 & $-0,359$ \\
\hline 4. Extrínseca - identificação & & & & $(0,74)$ & 0,220 & 0,325 & $-0,339$ \\
\hline 5. Extrínseca - introjeção & & & & & $(0,85)$ & 0,494 & 0,066 \\
\hline 6. Extrínseca - regulação externa & & & & & & $(0,83)$ & 0,047 \\
\hline 7. Desmotivação & & & & & & & $(0,71)$ \\
\hline Média & 6,00 & 4,99 & 4,88 & 6,22 & 3,47 & 4,43 & 1,41 \\
\hline Desvio padrão & 0,87 & 1,27 & 1,11 & 0,83 & 1,55 & 1,45 & 0,65 \\
\hline
\end{tabular}

Nível crítico de $\mathrm{r}=0,100 \mathrm{P}<0,05$.

A Tabela 2 revela o grau de estabilidade temporal de indicadores seletivos da EMA, mediante medidas emparelhadas obtidas com intervalo de 12 meses entre a primeira e a segunda medida, no subconjunto aleatório de 108 dentre os 285 participantes do estudo. 


\section{TABELA 2}

Diferenças e correlações entre medidas emparelhadas de indicadores da Escala de Motivação Acadêmica (EMA), obtidas com intervalo de 2 semestres $(\mathrm{N}=108)$

\begin{tabular}{lccccccc}
\hline & Medidas & & \multicolumn{2}{c}{ Diferença } & \multicolumn{3}{c}{ Correlação } \\
Indicadores da EMA & $1^{\mathrm{a}}$ & $2^{\mathrm{a}}$ & $\left(2^{\mathrm{a}}\right)-\left(1^{\mathrm{a}}\right)$ & $\mathrm{P}$ & $\mathrm{r}$ & $\mathrm{P}$ \\
\hline Desmotivação & 1,46 & 1,41 & $-0,05$ & 0,468 & 0,290 & 0,003 \\
Motivação intrínseca & 5,18 & 5,19 & 0,01 & 0,955 & 0,592 & $<0,001$ \\
Índice de & 9,72 & 9,67 & $-0,05$ & 0,847 & 0,544 & $<0,001$ \\
autodeterminação & & & & & & \\
Motivação autônoma & 5,71 & 5,77 & 0,06 & 0,316 & 0,625 & $<0,001$ \\
Motivação controlada & 3,97 & 4,25 & 0,28 & 0,015 & 0,535 & $<0,001$ \\
Motivação total & 4,96 & 5,07 & 0,12 & 0,093 & 0,612 & $<0,001$ \\
\hline
\end{tabular}

Dentre seis indicadores da EMA - motivação intrínseca, ou autônoma, ou controlada, ou total, desmotivação e índice de autodeterminação da motivação - houve diferença significante apenas entre as médias para motivação controlada, mais elevada na segunda medida. As correlações entre as medidas emparelhadas dos seis indicadores foram significantes em todos os casos, mas variando em magnitude, da menor para desmotivação $(r=$ 0,290; $\mathrm{P}=0,003)$ até a maior para motivação autônoma $(\mathrm{r}=0,625 ; \mathrm{P}<0,001)$.

A Tabela 3 mostra as correlações entre sete componentes da EMA e três medidas sucessivas do índice de rendimento acadêmico: ao término do $2^{\circ}$ semestre (referente ao primeiro ano do curso), antes da aplicação do instrumento; e, prospectivamente, ao término do $6^{\circ}$ e do $9^{\circ}$ semestres. Correlações significantes e positivas, mas de pequena magnitude ( $\mathrm{r}$ $<0,20)$ foram observadas entre rendimento acadêmico e motivação para saber e para vivenciar estímulos. As correlações prospectivas foram praticamente estáveis e mais fortes do que as retrospectivas. Na análise de regressão múltipla (stepwise), incluindo os sete componentes da EMA, o fator preditivo singular foi motivação para vivenciar estímulos (coeficiente de determinação $\mathrm{R}^{2}=0,026 \mathrm{~F}=7,6 \mathrm{P}=0,006$ ) - ao término do $9^{\circ}$ semestre, para exemplificar. 


\section{TABELA 3}

Coeficientes de correlação (r) entre componentes da Escala de Motivação Acadêmica (EMA) e índices de rendimento acadêmico cumulativo (IRA) ao término de três etapas $(\mathrm{N}=285)$

\begin{tabular}{lccc}
\hline & IRA $2^{\circ}$ semestre & IRA $6^{\circ}$ semestre & IRA $9^{\circ}$ semestre \\
Componentes da EMA & $\mathrm{r}$ & $\mathrm{r}$ & $\mathrm{r}$ \\
\hline Intrínseca - saber & 0,087 & 0,122 & 0,120 \\
Intrínseca - realização & 0,060 & 0,089 & 0,092 \\
Intrínseca - estímulo & 0,156 & 0,164 & 0,162 \\
Extrínseca - identificação & 0,069 & 0,084 & 0,103 \\
Extrínseca - introjeção & $-0,016$ & $-0,010$ & $-0,008$ \\
Extrínseca - regulação externa & 0,036 & 0,033 & 0,035 \\
Desmotivação & $-0,066$ & $-0,081$ & $-0,081$ \\
\hline
\end{tabular}

Nível crítico de $\mathrm{r}=0,100 \mathrm{P}=0,05$.

A Tabela 4 mostra as correlações entre os componentes da EMA e o índice de monitorias realizadas ao término do $6^{\circ}$, do $9^{\circ}$ e do $12^{\circ}$ semestres do curso. Correlações positivas e significantes, mas de pequena magnitude, foram observadas entre os três componentes de motivação intrínseca - bem como motivação extrínseca por regulação externa - e o número cumulativo de monitorias ao cabo das três etapas referidas. A relação mais forte do exercício da monitoria se deu com motivação para realização, ao término do sexto semestre $(\mathrm{r}=0,228)$. Na análise de regressão múltipla (stepwise), dois fatores preditivos positivos foram identificados: motivação para realização e regulação externa (coeficiente de determinação $\mathrm{R}^{2}=0,081 \mathrm{~F}=7,4 \mathrm{P}=0,001$ ) - ao término do $12^{\circ}$ semestre, para exemplificar. 


\section{TABELA 4}

Coeficientes de correlação (r) entre componentes da Escala de Motivação Acadêmica (EMA) e número de monitorias realizadas em disciplinas ao término do $6^{\circ}, 9^{\circ}$, e $12^{\circ}$ semestres do curso $(\mathrm{N}=$ 285)

\begin{tabular}{lccc}
\hline & $6^{\circ}$ semestre & $9^{\circ}$ semestre & $12^{\circ}$ semestre \\
Componentes da EMA & $\mathrm{r}$ & $\mathrm{r}$ & $\mathrm{r}$ \\
\hline Intrínseca - saber & 0,160 & 0,149 & 0,121 \\
Intrínseca - realização & 0,228 & 0,220 & 0,185 \\
Intrínseca - estímulo & 0,222 & 0,159 & 0,151 \\
Extrínseca - identificação & 0,023 & 0,006 & $-0,027$ \\
Extrínseca - introjeção & 0,114 & 0,075 & 0,063 \\
Extrínseca - regulação externa & 0,172 & 0,160 & 0,169 \\
Desmotivação & $-0,098$ & $-0,061$ & $-0,059$ \\
\hline
\end{tabular}

Nível crítico de $\mathrm{r}=0,098 \mathrm{P}=0,05$.

No conjunto total de participantes, o procedimento $K$-cluster de análise classificatória configurou quatro grupos distintos - doravante denominados A, B, C e D constituídos de 86, 111, 59 e 29 estudantes, respectivamente. Participantes nesses grupos diferiram significantemente entre si no índice da escala analógica de intenção de concluir o curso (análise de variância univariada, $\mathrm{F}=23,3 \mathrm{P}<0,001$; eta ${ }^{2}=0,199$ ). Os quatro grupos foram replicados em segunda análise classificatória com o subconjunto de participantes $(\mathrm{N}=$ 108). Observou-se, contudo, modesta força de associação (Cramér $\mathrm{V}=0,306 \mathrm{P}<0,001$ ) na classificação dos participantes entre a primeira e a segunda medida, no intervalo de 12 meses.

A Tabela 5 mostra as relações entre indicadores seletivos da EMA e os quatro grupos de participantes identificados na análise classificatória segundo o perfil motivacional. Análises de variância (univariada) revelaram diferenças muito significantes $(\mathrm{P}<0,001)$ entre os grupos, observando-se maior magnitude de efeito (eta $\left.{ }^{2}\right)$ para indicadores combinados: índice de autodeterminação ( eta $\left.^{2}=0,574\right)$, motivação autônoma ( $\left.\operatorname{eta}^{2}=0,635\right)$, motivação controlada $\left(\right.$ eta $\left.^{2}=0,714\right)$ e motivação total $\left(\right.$ eta $\left.^{2}=0,713\right)$. A maioria $(69,1 \%)$ dos participantes se distribuiu entre os grupos A e B, caracterizados por maior índice de autodeterminação da motivação e diferindo entre si principalmente em temos de motivação autônoma (maior para A), motivação controlada (menor para B) e, portanto, de motivação total (maior para A). Os dois grupos minoritários (C e D) - caracterizados por menor índice de autodeterminação da 
motivação - também diferiram em motivação autônoma (maior para $\mathbf{C}$ ), motivação controlada (menor para D) e, portanto, de motivação total (maior para $\mathbf{C}$ ).

\section{TABELA 5}

Comparações de indicadores de motivação entre grupos identificados por análise classificatória $(K$ means). Análise de variância univariada $(\mathrm{N}=285)$

\begin{tabular}{|c|c|c|c|c|c|c|c|}
\hline & $\underline{\text { Grupos }}$ & & & & & & \\
\hline Indicadores & $\mathbf{A}(\mathrm{n}=86)$ & $\begin{array}{c}\text { B } \\
(\mathrm{n}=111)\end{array}$ & $C(n=59)$ & $\begin{array}{c}\text { D } \\
(n=29)\end{array}$ & $\mathrm{F}$ & $\mathrm{P}$ & $\mathrm{Eta}^{2}$ \\
\hline Motivação autônoma & 6,34 & 5,87 & 5,39 & 4,32 & 163,11 & $<0,001$ & 0,635 \\
\hline Motivação controlada & 5,32 & 3,02 & 4,47 & 2,40 & 233,70 & $<0,001$ & 0,714 \\
\hline Motivação total & 5,90 & 4,75 & 4,86 & 3,58 & 233,01 & $<0,001$ & 0,713 \\
\hline $\begin{array}{l}\text { Índice de } \\
\text { autodeterminação }\end{array}$ & 11,02 & 11,87 & 6,94 & 6,24 & 126,00 & $<0,001$ & 0,574 \\
\hline Motivação intrínseca & 6,03 & 5,35 & 4,71 & 4,02 & 83,67 & $<0,001$ & 0,472 \\
\hline $\begin{array}{l}\text { Motivação - } \\
\text { identificação }\end{array}$ & 6,64 & 6,38 & 6,08 & 4,61 & 88,98 & $<0,001$ & 0,487 \\
\hline Desmotivação & 1,19 & 1,09 & 2,05 & 2,01 & 66,42 & $<0,001$ & 0,415 \\
\hline
\end{tabular}

Não houve diferença significante no índice de rendimento acadêmico entre os quatro grupos de perfil motivacional, exceto quando agrupados, segundo o índice de autodeterminação da motivação, em dois subconjuntos, o de elevada $(\mathbf{A}+\mathbf{B})$ e o de baixa $(\mathbf{C}+\mathbf{D})$ autodeterminação. O rendimento acadêmico foi maior no subconjunto $(\mathbf{A}+\mathbf{B})$ e a diferença entre os subconjuntos, que não era significante ao término do $2^{\circ}$ semestre $(F=2,35$ $\mathrm{P}=0,127)$, ficou significante ao término do $6^{\circ}$ semestre $(\mathrm{F}=3,97 \mathrm{P}=0,047)$ e do $9^{\circ}$ semestre $(\mathrm{F}=4,24 \mathrm{P}=0,040)$.

Em contraposição, ocorreram diferenças significantes entre os quatro grupos no índice de monitoria, em três etapas da graduação, conforme resumido na Tabela 6. 


\section{TABELA 6}

Comparações do índice de monitorias realizadas ao término de três etapas do curso entre grupos identificados por análise classificatória (K-means). Análise de variância univariada $(\mathrm{N}=285)$

\begin{tabular}{lccccccc}
\hline & Grupos & & & \multicolumn{3}{c}{ Análise } \\
Etapa & $\mathbf{A}(\mathrm{n}=86)$ & $\mathbf{B}(\mathrm{n}=$ & $\mathbf{C ~}(\mathrm{n}=89)$ & $\mathbf{D}(\mathrm{n}=29)$ & F teste & $\mathrm{P}$ & $\mathrm{Eta}^{2}$ \\
& & $111)$ & & & & & \\
\hline $6^{\mathbf{0}}$ semestre & 2,50 & 2,06 & 1,95 & 1,66 & 3,94 & 0,009 & 0,040 \\
$9^{\mathbf{0}}$ semestre & 4,14 & 3,69 & 3,49 & 2,90 & 3,46 & 0.017 & 0,036 \\
$12^{\circ}$ semestre & 4,78 & 4,19 & 4,03 & 3,48 & 2,83 & 0,039 & 0,029 \\
\hline
\end{tabular}

O índice de monitoria mostrou valor decrescente do grupo A ao grupo D - isto é, no mesmo sentido do decréscimo em motivação autônoma. A magnitude do efeito foi pequena e decrescente entre o $6^{\circ}\left(\mathrm{eta}^{2}=0,040\right)$, o $9^{\circ}\left(\right.$ eta $\left.^{2}=0,036\right)$, e o $12^{\circ}\left(\right.$ eta $\left.^{2}=0,029\right)$ semestre do curso. Entretanto, a diferença no índice de monitoria entre os grupos extremos de motivação autônoma (A e D) permaneceu significante ao longo das três etapas.

Ressalve-se, ainda, que o efeito preditivo significante do perfil motivacional mostrou-se independente de sexo e do efeito, bem mais expressivo, do índice de rendimento acadêmico no índice de realização de monitoria. A Tabela 7 mostra os resultados da análise de variância univariada para índice de monitoria como variável de desfecho, grupo de perfil motivacional e sexo como fatores fixos e índice de rendimento como covariável - ao término do nono semestre do curso. Cerca de $3 \%\left(\operatorname{eta}^{2}=0,031\right)$ na variabilidade do índice de monitoria é atribuível ao efeito do perfil motivacional. 
TABELA 7

Efeitos (between-subjects) de grupos de análise classificatória (K-means), sexo e índice de rendimento acadêmico, no índice de monitoria ao término do $9^{\circ}$ semestre como variável de desfecho. Análise de variância univariada $(\mathrm{N}=285)$

\begin{tabular}{lrrrrrr}
\hline Fonte de variação & $\begin{array}{r}\text { Soma de } \\
\text { quadrados }\end{array}$ & df & $\begin{array}{r}\text { Média } \\
\text { (MS) }\end{array}$ & F & Significância & Eta $^{2}$ \\
& (SQ) & & & & \\
& 237,21 & 5 & 47,44 & 16,02 & $<0,001$ & 0,223 \\
\hline Modelo corrigido & 26,69 & 3 & 8,90 & 3,00 & 0,031 & 0,031 \\
Grupos & 2,38 & 1 & 2,38 & 0,80 & 0,371 & 0,003 \\
Sexo & 197,46 & 1 & 197,46 & 66,69 & $<0,001$ & 0,193 \\
Rendimento acadêmico & 86,09 & 1 & 86,09 & 29,08 & $<0,001$ & 0,094 \\
Intercepto & 826,03 & 279 & 2,96 & & & \\
Erro & 4976,00 & 285 & & & & \\
Total & 1063,24 & 284 & & & & \\
Total corrigido & & & & & & \\
\hline
\end{tabular}

\section{DISCUSSÃO}

Os componentes da EMA que caracterizaram predominantemente a orientação motivacional dos estudantes de medicina, no início do segundo ano, foram motivação por identificação e motivação para saber - os quais expressariam, respectivamente, a importância pessoal e o interesse que são atribuídos ao aprendizado no curso. Esses elementos possivelmente refletem a adaptação motivacional à ambiência acadêmica, na transição entre o ensino médio e o ensino superior - conforme observado noutro contexto por Koestner e Losier (2002, p.115) - e são congruentes com os níveis de valoração da vivência inicial indicados pelos mesmos estudantes.

Os resultados das medidas emparelhadas - no subconjunto de 108 participantes mostraram relativa estabilidade, em médio prazo, das respostas contidas nos indicadores de motivação, isto é, os componentes simples e combinados da EMA. O nível e o grau de estabilidade da motivação acadêmica, primariamente autônoma, são congruentes com a massiva adesão ao curso, que foi expressa no baixo índice de evasão por mudança de opção profissional (2,0\%, seis desistências), entre os 296 estudantes incluídos no estudo. Tais achados sugerem que o padrão de motivação educacional, na esfera contextual, reflete 
parcialmente a orientação global do indivíduo, decorrente de características psicológicas relativamente estáveis. Resultados de análise recente de motivação e personalidade em estudantes de medicina reforçam essa noção (TANAKA et al., 2009). Esses autores mostraram associação expressiva e significante entre motivação acadêmica intrínseca e traços de personalidade - a saber, persistência na dimensão de temperamento e autonomia e autotranscendência na dimensão de caráter - que foram identificados mediante aplicação do Inventário de Temperamento e Caráter (CLONINGER et al., 1993).

Os dados da reaplicação da EMA no presente estudo sugerem, entretanto, alguma mudança no padrão motivacional dos estudantes, na passagem da ambiência de ensino básico - na primeira medida - para a ambiência clínica, na segunda medida. Essa passagem ou transição implica mudanças multidimensionais na ambiência, em termos físicos, semânticos e de compromisso (KOENS et al., 2005). Aparentemente, motivos instrumentais tornaram-se mais significativos na transição para a ambiência clínica, seja por influências situacionais ou adaptação motivacional aos requisitos da formação clínica. Segundo Patrick e Williams (2009), a sobrecarga de informação e emoção na iniciação clínica tradicional dificulta a integração plena das vivências intelectuais e afetivas pelos aprendizes. Em termos da teoria da autodeterminação, esses e outros fatores da ambiência restringiriam a satisfação funcional das necessidades básicas de autonomia, competência e vínculo - diminuindo, portanto, o alcance do potencial de motivação autônoma ou compromisso autodeterminado dos estudantes.

As diferenças significativas nas relações entre os indicadores básicos da EMA e os desfechos de rendimento acadêmico e de exercício de monitoria são compatíveis com interrelações entre fatores pessoais e situacionais e robustecem a caracterização multidimensional das respostas ao instrumento entre estudantes brasileiros. Tais diferenças envolveram a abrangência dos tipos de motivação, a sustentabilidade ou estabilidade temporal do efeito e a força ou magnitude da associação. A correlação entre a motivação autônoma inicial e o rendimento acadêmico cumulativo foi mínima, embora significante e aparentemente estável. O achado sugere que o padrão de motivação, caracterizado em nível contextual no início da graduação, tem efeito previsível no nível de rendimento, mas influência insignificante na mudança de rendimento ao longo do curso.

Diferentemente, o efeito preditivo do padrão de motivação no índice de atividade de monitoria foi mais expressivo na força de associação e complexo: incluiu os três tipos de 
motivação intrínseca, com predomínio de motivação para realização e tendência temporal decrescente; além disso, abrangeu o componente de motivação extrínseca por regulação externa com tendência temporal estável. Esses achados ilustram a situação de duplo propósito na perspectiva da atividade estudantil - investigada por Vansteenkiste et al. (2009) - na medida em que o exercício eletivo da monitoria proporcionaria maior grau de liberdade no aprofundamento de matérias de interesse, mas também propiciaria recompensas curriculares, inclusive quanto à motivação vocacional do participante. Nessa concepção, os dados da participação iterativa na monitoria implicam fatores ou requisitos instrumentais na antevisão do acesso à prática profissional, mas não descartam o potencial de blindagem das percepções de autonomia, competência e vínculo, resultante da comunicação educativa dos monitores com seus pares menos experientes. O ponto central nessa interpretação reside na noção que as pessoas podem endossar simultaneamente formas de motivação com maior (autônoma) e menor (controlada) autodeterminação ao se engajar numa atividade duradoura e relevante (VALLERAND et al., 2008, p.259).

O significado prático dessa noção foi realçado pelos dados da análise classificatória, os quais identificaram quatro configurações motivacionais entre os participantes, em termos da magnitude relativa de motivação autônoma e controlada. À semelhança do estudo de Ratelle et al. (2007) com estudantes do ensino superior verificou-se no conjunto de participantes a presença de dois grupos distintos com nível elevado de motivação autônoma, mas diferindo significantemente no nível de motivação controlada. Esses dois grupos mostraram resultados equivalentes nos indicadores de progresso acadêmico, seja em termos de rendimento na aprendizagem ou, particularmente, na realização da atividade eletiva de monitoria.

Registre-se que a maioria dos participantes expressou nível expressivo de motivação autônoma, embora menos de um terço tenha configurado o perfil motivacional nítido de autodeterminação (alta motivação autônoma, baixa motivação controlada) ao iniciar o segundo ano da formação médica. Aparentemente, essa proporção não se alterou substancialmente na transição para a ambiência clínica - ainda que, infelizmente, a precisão dos dados tenha sido fragilizada na replicação da análise classificatória na minoria (38\%) do total de participantes. 
Os resultados apresentados confirmam e estendem achados de trabalhos anteriores, que mostravam a diversidade e a inter-relação dialética de fatores pessoais e contextuais em conexão com o espectro de autodeterminação da motivação dos estudantes de medicina (SOBRAL, 2008). Os achados são consistentes com o modelo teórico e, portanto, dão suporte para o entendimento da configuração de motivação educacional na ambiência intrincada da formação médica. Implicam a questão do ajuste da ambiência de aprendizagem aos diversos perfis motivacionais - seja no domínio educacional ou vocacional - mas visando sobretudo ao fortalecimento da autodeterminação dos aprendizes na perspectiva da missão humanista da educação médica. Além disso, sugerem a hipótese que motivação educacional e motivação vocacional ou de trabalho sejam parcialmente concordantes ou discordantes, em grupos distintos de estudantes de medicina.

O estudo tem várias limitações, entre as quais a proveniência dos dados - restrita a uma única instituição - e o uso primordial de questionário de auto-relato para aferir motivação. Outra limitação foi inexistência de avaliação múltipla, com procedimentos estatísticos sofisticados - do espectro motivacional ao longo do período de acompanhamento dos participantes, gerando incerteza na interpretação da estabilidade do perfil de motivação. De qualquer forma, análises de sequências causais, integrando determinantes e desfechos motivacionais, requerem procedimentos de modelagem por equações estruturais indisponíveis no pacote estatístico utilizado - conforme indicado por Guay e Vallerand (1997), entre outros.

As limitações do estudo, por outro lado, reforçam o pleito de Guay et al. (2008) para pesquisa direcionada à averiguação de proposições específicas relacionadas à teoria da autodeterminação, no campo da educação. Dentre as várias recomendações propostas por aqueles investigadores, o presente autor realça a necessidade de estudos longitudinais com múltiplos pontos de coleta de dados e na perspectiva de pesquisa centrada em grupos. 
Os resultados, em conclusão, sugerem que indicadores de motivação caracterizados no início da graduação têm efeito preditivo pequeno, mas significante no progresso acadêmico dos estudantes, em termos de desempenho na aprendizagem e consecução de monitorias em disciplinas da formação profissional. Os achados sugerem também que a configuração da motivação importa: estudantes que endossam mais definidamente orientação motivacional autônoma - associada ou não à motivação controlada têm mais sucesso na busca de monitoria e persistência no exercício dessa atividade junto aos seus pares.

\section{REFERÊNCIAS}

CLATWORTHY, J.; BUICK, D.; HANKINS, M.; WEINMAN, J.; HORNE, R. The use and reporting of cluster analysis in health psychology: A review. British Journal of Health Psychology, v. 10, n. 3, p.329-358, 2005.

CLONINGER, C. R.; SVRAKIC, D. M.; PRZYBECK, T. R. A psychobiological model of temperament and character. Archives of General Psychiatry, v. 50, n. 12, p.975-990, 1993.

DECI, E. L.; RYAN, R. M. A motivational approach to self: integration in personality. In: DIENSTBIER, R. (Org.). Nebraska symposium on motivation. Lincoln, NE: University of Nebraska Press, 1991. p.237-288. (v. 38 - Perspectives on motivation).

DECI, E. L.; RYAN, R. M. Facilitating optimal motivation and psychological well-being across life's domains. Canadian Psychology, v. 49, n. 1, p.14-23, 2008.

DECI, E. L.; VALLERAND, R. J.; PELLETIER, L. G.; RYAN, R. M. Motivation in education: the self-determination perspective. Educational Psychologist, v. 26, n. 3/4, p.325346, 1991.

DECI, E. L.; RYAN, R. M.; WILLIAMS, G. C. Need satisfaction and the self-regulation of learning. Learning and Individual Differences, v. 8, n. 3, p.165-183, 1996.

DECI, E. L.; RYAN, R. M. The "what" and "why" of goal pursuits: human needs and the selfdetermination of behavior. Psychological Inquiry, v.11, n. 4, p.227-268, 2000.

FAIRCHILD, A. J.; HORST, S. J.; FINNEY, S. J.; BARRON, K. E. Evaluating existing and new validity evidence for the Academic Motivation Scale. Contemporary Educational Psychology, v. 30, n. 3, p.331-358, 2005. 
GUAY, F.; MAGEAU, G. A.; VALLERAND, R. J. On the hierarchical structure of selfdetermined motivation: A test of top-down, bottom-up, reciprocal, and horizontal effects. Personality and Social Psychology Bulletin, v. 29, n. 8, p.992-1004, 2003.

GUAY, F.; RATELLE, C. F.; CHANAL, J. Optimal learning in optimal contexts: the role of self-determination in education. Canadian Psychology, v. 49, n. 1, p.233-240, 2008.

GUAY, F.; VALLERAND, R. J. Social context, students' motivation, and academic achievement: Toward a process model. Social Psychology of Education, v.1, n. 3, p.211-233, 1996.

HOWELL, D. C. Statistical Methods for Psychology. 5. ed. Pacific Grove, CA: Duxbury, 2002.

KOENS, F.; MANN, K. V.; CUSTERS, E. J. F. M.; TEN CATE, O. T. Analysing the concept of context in medical education. Medical Education, v. 39, n. 12, p.1243-1249, 2005.

KOESTNER, R.; LOSIER, G. F. Distinguishing three ways of being internally motivated: A closer look at introjection, identification, and intrinsic motivation. In: DECI, E. L.; RYAN, R. M. (Org.). Handbook of self-determination research. Rochester: University of Rochester Press, 2002, p.101-121.

MANN, K. V. Motivation and medical education: how theory can inform our practice. Academic Medicine, v. 74, n. 3, p.237-239, 1999.

MISCH, D. A. Andragogy and medical education: Are medical students internally motivated to learn? Advances in Health Sciences Education, v. 7, n. 2, p.153-160, 2002.

PATRICK, H.; WILLIAMS, G. C. Self-determination in medical education. Encouraging medical educators to be more like blues artists and poets. Theory and Research in Education, v. 7, n. 2, p.184-193, 2009.

RATELLE, C. F.; GUAY, F.; VALLERAND, R. J.; LAROSE, S.; SENÉCAL, C. Autonomous, controlled, and amotivated types of academic motivation: a person-oriented analysis. Journal of Educational Psychology, v. 99, n. 4, p.734-746, 2007.

REEVE, J.; DECI, E. L.; RYAN, R. M. Self-determination theory: a dialectical framework for understanding socio-cultural influences on student motivation. In: VAN ETTEN, S.; PRESSLEY, M. (Org.). Big Theories Revisited. Greenwich, CT: Information Age Press, 2004, p.31-60.

RYAN, R. M.; DECI, E. L. Intrinsic and extrinsic motivations: classic definitions and new directions. Contemporary Educational Psychology, v. 25, n. 1, p.54-67, 2000.

SOBRAL, D. T. Autodeterminação da motivação em alunos de Medicina: relação com motivos de escolha da opção e intenção de adesão ao curso. Revista Brasileira de Educação Médica, v. 32, n. 1, p.56-65, 2008. 
. Espectro de motivação do aprendiz de medicina: uso da escala de motivação acadêmica. Psicologia Teoria e Pesquisa, v. 19, n. 1, p.25-31, 2003.

. What kind of motivation drives medical students learning quests? Medical

Education, v. 38, n. 9, p.950-957, 2004.

TABACHNICK, B. G.; FIDELL, L. S. Using multivariate statistics. 5 ed. Boston: Pearson, 2007.

TANAKA, M.; MIZUNO, K.; FUKUDA, S.; TAJIMA, S.; WATANABE, Y. Personality traits associated with intrinsic motivation in medical students. Medical Education, v. 43, n. 4, p.384-387, 2009.

VALLERAND, R. J. Toward a hierarchical model of intrinsic and extrinsic motivation. In: ZANNA, M. P. (Ed.), Advances in Experimental Social Psychology, v. 29. San Diego: Academic Press, 1997, p.271-360.

VALLERAND, R. J.; BLAIS, M. R.; BRIERE, N. M.; PELLETIER, L. G. Construction et validation de l'Echelle de Motivation en Education. Canadian Journal of Behavioural Sciences, v. 21, n. 3, p.323-349, 1989.

VALLERAND, R. J.; RATELLE, C. F. Intrinsic and extrinsic motivation: a hierarchical model. In: DECI, E.L.; RYAN, R.M. (Org.). Handbook of self-determination research. Rochester: University of Rochester Press, 2002, p.37-64.

VALLERAND, R. J.; PELLETIER, L. G.; BLAIS, M. R.; BRIÈRE, N. M.; SENÉCAL, C.; VALLIÈRES, E. F. On the assessment of intrinsic, extrinsic and amotivation in education: evidence on the concurrent and construct validity of the Academic Motivation Scale.

Educational and Psychological Measurement, v. 53, n. 1, p.159-172, 1993.

VALLERAND, R. J.; PELLETIER, L. G.; KOESTNER, R. Reflections on self-determination theory. Canadian Psychology, v. 49, n. 1, p.257-262, 2008.

VALLERAND, R. J.; PELLETIER, L. G.; BLAIS, M. R.; BRIÈRE, N. M.; SENÉCAL, C.; VALLIÈRES, E. F. The Academic Motivation Scale: a measure of intrinsic, extrinsic, and amotivation in education. Educational and Psychological Measurement, v. 52, n. 4, p.10031017, 1992.

VANSTEENKISTE, M.; SOENENS, B.; VERSTUYF, J.; LENS, W. 'What is the usefulness of your schoolwork?: The differential effects of intrinsic and extrinsic goal framing on optima learning. Theory and Research in Education, v. 7, n. 2, p.155-163, 2009.

WILLIANS, G. C.; DECI, E. L. Internalization of biopsychosocial values by medical students: a test of self-determination theory. Journal of Personality and Social Psychology, v. 70, n. 4, p.767-779, 1996.

;

Internal Medicine, v. 129, n. 4, p.303-308, 1998. 


\begin{abstract}
Agradecimentos
$\mathrm{O}$ autor registra o apoio institucional, agradece a generosa cooperação e o interesse real dos estudantes participantes e expressa seu reconhecimento ao Dr. Robert J. Vallerand por fornecer o instrumento original e concordar com a divulgação dos resultados da linha de estudos.
\end{abstract}

DEJANO TAVARES SOBRAL

Universidade de Brasília - Faculdade de Medicina C.P. 04569 - 70919-970 Brasília - DF Médico, Residência em Clínica Médica (Faculdade de Medicina, UFRJ) Especialista em Medicina Interna (Conselho Regional de Medicina DF) Diploma in Medical Education (University of Dundee) Membro do Conselho Científico (Revista Brasileira de Educação Médica)

Professor Emérito (Universidade de Brasília) Tel.: 3307-2269 / 3577-2268/ Fax: 3577-35581 E-mail: dtsobral@unb.br/dtsobral@netscape.net

Recebido em: 20/07/2009 Publicado em: 30/10/2009 